\title{
BOUNDS AND QUOTIENT ACTIONS OF INNATELY TRANSITIVE GROUPS
}

\author{
JOHN BAMBERG
}

(Received 17 December 2003; revised 31 March 2004)

\author{
Communicated by E. A. O'Brien
}

\begin{abstract}
Finite innately transitive permutation groups include all finite quasiprimitive and primitive permutation groups. In this paper, results in the theory of quasiprimitive and primitive groups are generalised to innately transitive groups, and in particular, we extend results of Praeger and Shalev. Thus we show that innately transitive groups possess parameter bounds which are similar to those for primitive groups. We also classify the innately transitive types of quotient actions of innately transitive groups.
\end{abstract}

2000 Mathematics subject classification: primary 20B05, 20B 14; secondary $20 \mathrm{~B} 35$.

\section{Introduction}

A finite permutation group $G$ acting on a set $\Omega$ is said to be innately transitive if it contains a transitive minimal normal subgroup. We call such a subgroup a plinth for $G$. Now $G$ can have at most two transitive minimal normal subgroups (see [1, Lemma 5.1]), and if it has two, then there is an element in the normaliser of $G$ that interchanges them. So up to permutational isomorphism, any of the at most two minimal normal subgroups of $G$ can be taken to be the plinth of $G$. Innately transitive groups have been studied in depth by the author in work coauthored with Praeger [1], and their interest and application is outlined in the introduction of [1].

Let $G$ be a group acting on a set $\Omega$, and suppose $\mathscr{B}$ is a $G$-invariant partition of $\Omega$. Then the action of $G$ on $\mathscr{B}$ is called a quotient action of $G$. A quotient action

The author would like to thank Prof. Cheryl E. Praeger, for her guidance and advice on this paper, and Assoc/Prof. Tim Penttila for many discussions on some of the material in this paper. Special thanks go to Dr. Michael Giudici for reading and giving advice on drafts of this paper.

(C) 2005 Australian Mathematical Society 1446-7887/05 $\$$ A2.00+0.00 
is essentially the image of a permutational transformation, which is an 'intertwiner' between two group actions and exists categorically as the natural weakening of a permutational isomorphism. Similar objects have been widely studied in homological algebra and representation theory, and they provide a more general context for results on quotient actions.

A transitive permutation group $G$ on a finite set $\Omega$ is imprimitive if there exists a nontrivial $G$-invariant partition $\mathscr{B}$ of $\Omega$ (that is, $1<|\mathscr{B}|<|\Omega|$ ). A transitive permutation group is primitive if it is not imprimitive. Every nontrivial normal subgroup of a primitive group is transitive (see [3, Theorem 1.6A]), but the converse is not true. A finite permutation group $G$ is called quasiprimitive if every non-trivial normal subgroup of $G$ is transitive. Every primitive group is quasiprimitive, and every quasiprimitive group is innately transitive. The similarity in the structure theorems for primitive groups (see [5]), quasiprimitive groups (see [6]), and innately transitive groups (see [1]) indicate that results on the properties of primitive groups might also be true for quasiprimitive and innately transitive groups. However, a quasiprimitive group has at most two minimal normal subgroups, but for an arbitrary integer $k$, there exist innately transitive groups which have at least $k$ minimal normal subgroups. Recently, Heath-Brown, Praeger, and Shalev [4] have proved that for most positive integers $n$, the only finite innately transitive permutation groups of degree $n$ are $A_{n}$ and $S_{n}$ in their natural action (which are primitive groups).

It was the study of properties of quasiprimitive groups by Praeger and Shalev (see [10]) that motivated this paper. Their goal was to generalise classical results about finite primitive permutation groups to quasiprimitive ones. Our goal is similar, in that we lift results on quasiprimitive groups to the context of innately transitive groups. Given an imprimitive quasiprimitive group $G$ acting on a finite set $\Omega$, there exists a maximal $G$-invariant partition $\mathscr{B}$ of $\Omega$. The finite permutation group $G^{\mathscr{B}}$ induced by the action of $G$ on $\mathscr{B}$ is primitive and isomorphic to $G$. Praeger and Shalev's technique, in almost all of their results, was to analyse this special type of quotient action and transfer classical results about primitive groups to quasiprimitive groups via this natural correspondence. In this paper, we find that a similar technique can be used for innately transitive groups. If $G$ is innately transitive but non-quasiprimitive, the permutation group induced by the action of $G$ on the orbits of the centraliser of the plinth is quasiprimitive. Hence we can transfer some results on quasiprimitive groups to innately transitive groups via this correspondence.

In this paper, we analyse bounds, innately transitive types, and other properties of innately transitive groups. We already know from [1] some useful properties of innately transitive groups.

LEMMA 1.1. Let $G$ be an innately transitive group on a set $\Omega$ with plinth $K$, and let $\alpha \in \Omega$. Then we have the following: 
(1) $G$ is quasiprimitive if and only if $\mathrm{C}_{G}(K)=1$ or $\mathrm{C}_{G}(K)$ is transitive.

(2) $K_{\alpha}$ is a normal subgroup of the setwise stabiliser $K_{\Delta}$ in $K$ of the $\mathrm{C}_{G}(K)$-orbit $\Delta=\alpha^{\mathrm{C}_{G}(K)}$, and $K_{\Delta} / K_{\alpha} \cong \mathrm{C}_{G}(K)$.

(3) If $K_{\alpha}$ is a subdirect subgroup of $K$, and $K$ is nonabelian and nonsimple, then $G$ is quasiprimitive.

(4) The group $G^{\mathscr{C}}$ induced by the action of $G$ on the set of $\mathrm{C}_{G}(K)$-orbits in $\Omega$ is quasiprimitive with kernel $\mathrm{C}_{G}(K), K$ acts faithfully, and the permutation group $K^{\mathscr{C}}$ induced by the action of $K$ is the plinth of $G^{\mathscr{C}}$.

$\mathrm{C}_{G}(K)$ is semiregular.

It is also true that if $\mathrm{C}_{G}(K)$ is transitive, then $G$ is primitive (see [1, Lemma 5.1]). The above properties are essentially properties of the quotient action of $G$ on the orbits of the centraliser of the plinth. Similar results also hold for abstract permutational transformations (see Section 5).

In the subsequent three sections, we give a brief account of the structure of innately transitive groups, we revise some background theory and conventions, and provide some examples of innately transitive groups which we use to prove later results. In Section 5, we give an expository summary of the definitions and elementary properties of permutational transformations. Our goal in Sections $5-8$ is to state and prove a theorem which encapsulates seven generalisations of results from [10]. Finally, in Section 9, we give a complete account of the innately transitive types of quotient actions of innately transitive groups, in a similar manner to that of Praeger's [7] investigation of quotient actions of quasiprimitive groups.

\section{The structure of innately transitive groups}

- The O'Nan-Scott Theorem (see [5]) is a result that partitions the finite primitive groups into eight disjoint types (see also [8]). Praeger's theorem for quasiprimitive groups is analogous to the O'Nan-Scott Theorem and partitions the finite quasiprimitive groups in a similar way to the case subdivision of finite primitive groups (see [8]). However the case subdivision used to describe innately transitive groups in [1] has more parts to the partition. The quasiprimitive type known traditionally as Product Action type, is generalised to contain innately transitive groups that are possibly not quasiprimitive. On top of this, there are three extra types defined: Almost Simple Quotient type, Product Quotient type and Diagonal Quotient type. Table 1 gives a summary of the subdivision of finite innately transitive groups.

Let $G$ be an innately transitive group with plinth $K$ acting on a set $\Omega$, let $\alpha \in \Omega$, and let $\Delta=\alpha^{\mathrm{C}_{G}(K)}$. Note that $K=T^{k}$ for some simple group $T$ and positive integer $k$. Then $G$ is of Abelian Plinth (AP), Holomorph of a Simple Group (HS), Almost Simple (AS), Almost Simple Quotient (ASQ), Holomorph of a Compound Group (HC), Twisted 
TABLE 1. A summary of the partition of finite innately transitive groups defined by certain properties.

\begin{tabular}{|c|c|c|c|c|c|c|c|c|c|c|}
\hline Property & AP & HS & AS & ASQ & HC & TW & PQ & DQ & PA & DT \\
\hline$K$ is abelian & $\checkmark$ & $\times$ & $\times$ & $\times$ & $\times$ & $\times$ & $\times$ & $\times$ & $\times$ & $\times$ \\
$K$ is simple & & $\checkmark$ & $\checkmark$ & $\checkmark$ & $\times$ & $\times$ & $\times$ & $\times$ & $\times$ & $\times$ \\
$K$ is regular & $\checkmark$ & $\checkmark$ & & & $\checkmark$ & $\checkmark$ & $\checkmark$ & $\checkmark$ & $\times$ & $\times$ \\
$C_{G}(K)=1$ & $\times$ & $\times$ & $\checkmark$ & $\times$ & $\times$ & $\checkmark$ & $\times$ & $\times$ & & $\checkmark$ \\
$C_{G}(K)$ is transitive & $\checkmark$ & $\checkmark$ & $\times$ & $\times$ & $\checkmark$ & $\times$ & $\times$ & $\times$ & $\times$ & $\times$ \\
$K_{\alpha}$ is subdirect in $K$ & $\times$ & & & & $\times$ & $\times$ & $\times$ & $\times$ & $\times$ & $\checkmark$ \\
$K_{\Delta}$ is subdirect in $K$ & $\checkmark$ & & & & $\checkmark$ & $\times$ & $\times$ & $\checkmark$ & $\times$ & $\checkmark$ \\
\hline
\end{tabular}

Wreath (TW), Product Quotient (PQ), Diagonal Quotient (DQ), Product Action (PA), or Diagonal (DT) type. Certain properties of these types are summarised in Table 1, and complete descriptions of the types are given in [1, Section 3]. We say that an innately transitive group $G$ with plinth $K$ is of $A S Q_{\text {reg }}$ type if $K$ is a nonabelian simple group acting regularly and $\mathrm{C}_{G}(K)$ is nontrivial and intransitive. We may sometimes refer to two subcases of the Diagonal type known as Simple Diagonal (SD) type and Compound Diagonal $(C D)$ type, which occur respectively when $K_{\alpha}$ is or is not a full diagonal subgroup of $K$. If $G$ is of Abelian Plinth, Holomorph of a Simple Group, or Holomorph of a Compound Group type, then $G$ is primitive.

\section{Some background theory}

In this section, we revise some standard background material. We will assume that the reader is familiar with the basics of permutation group theory, such as the well known fact that if $G$ acts transitively on a set $\Omega, \alpha$ is a point of $\Omega$, and $K$ is a transitive subgroup of $G$, then $G=K G_{\alpha}$ (see Dixon and Mortimer's book [3] for an introduction to permutation group theory). Throughout this paper, we will use the standard notation $G^{\Sigma}$ to mean the permutation group induced by the action of $G$ on $\Sigma$.

The holomorph of a group $X$ is the semidirect product $X \rtimes \operatorname{Aut}(X)$ and is denoted $\operatorname{Hol}(X)$. Furthermore, the normaliser of $X$ in $\operatorname{Sym}(X)$ is isomorphic to $\operatorname{Hol}(X)$, and $\mathrm{Hol}(X)$ acts naturally on $X$ in that $X$ acts on itself by right multiplication (see [1, Section 2]). Let $G$ be a finite innately transitive permutation group on a set $\Omega$ with plinth $K$, and suppose $K$ is regular. Then it is well-known (see [1, Section 2]) that $G$ is permutationally isomorphic to a subgroup of $\operatorname{Hol}(K)$ in its natural action on $K$.

Let $\prod_{i \in l} H_{i}$ be a direct product of groups. Given $j \in I$, we denote the natural projection map from $\prod_{i \in l} H_{i}$ to $H_{j}$ by $\pi_{j}$. A group $G$ is a subdirect product of $\prod_{i \in I} H_{i}$ if there is an embedding $\phi: G \rightarrow \prod_{i \in I} H_{i}$ such that $\phi \circ \pi_{j}: G \rightarrow H_{j}$ is an epimorphism for each $j \in I$. In the case that $G$ is a subgroup of $\prod_{i \in I} H_{i}$ and $\phi$ is the inclusion map, we say that $G$ is a subdirect subgroup of $\prod_{i \in l} H_{i}$. If $G$ is a subgroup 
of $\prod_{i \in I} H_{i}$, we say that $G$ is a diagonal subgroup of $\prod_{i \in I} H_{i}$ if the restriction of $\pi_{j}$ to $G$ is injective for each $j \in I$. We say that $G$ is a full diagonal subgroup of $\prod_{i \in I} H_{i}$ if $G$ is both a subdirect and diagonal subgroup of $\prod_{i \in I} H_{i}$. In this case, when $G$ is a full diagonal subgroup of $\prod_{i \in I} H_{i}$, the direct factors $H_{i}$ are isomorphic to a common group $H$ and $G \cong H$. If $I=\{1, \ldots, n\}$, then each full diagonal subgroup $G$ of $\prod_{i \in I} H_{i}$ is of the form $\left\{\left((g) \gamma_{1},(g) \gamma_{2}, \ldots,(g) \gamma_{n}\right): g \in G\right\}$ where for each $i \in I$, $\gamma_{i}$ is an isomorphism from $G$ onto $H_{i}$. For every group $H$ and integer $n$, the set $\{(h, h, \ldots, h): h \in H\}$ is a full diagonal subgroup of $H^{n}$ called the the straight diagonal subgroup of $H^{n}$, which we will denote by $\operatorname{Diag}\left(H^{n}\right)$.

A base of a permutation group $G \leq \operatorname{Sym}(\Omega)$ of degree $n$, is a subset of $\Omega$ that has a trivial pointwise stabiliser in $G$. Let $b_{\Sigma}(G)$ denote the minimal base size of $G$ acting on $\Sigma$. The minimal degree $m_{\Omega}(G)$ of a permutation group $G \leq \operatorname{Sym}(\Omega)$ is the minimum number of points moved by a nontrivial element of $G$.

\section{Some examples}

Here we give some examples of innately transitive groups which are not only useful in understanding the nature of innately transitive groups, but which will also serve as constructions for the proof of a result (Theorem 9.2) on the quotient actions of innately transitive groups.

EXAMPLE 1 (Product Quotient type (PQ)). Let $H$ be an innately transitive group of Almost Simple Quotient type with regular plinth $M$, and let $k>1$. Then $G=H$ wr $S_{k}$ is innately transitive in product action with regular plinth $K=M^{k}$. Moreover, $\mathrm{C}_{G}(K)=\left(\mathrm{C}_{H}(M)\right)^{k}$ and $\left|\mathrm{C}_{H}(M)\right|<|M|$, and hence $\mathrm{C}_{G}(K)$ is not a subdirect subgroup of the left regular representation of $K$. Therefore, $G$ is of Product Quotient type by [1, Remarks 10.2 and Proposition 10.3].

EXAMPLE 2 (Diagonal Quotient type (DQ)). Let $T$ be a nonabelian simple group, let $k>1$, let $K=T^{k}$, let $m$ be a proper divisor of $k$, let $A$ be the straight diagonal subgroup of $\operatorname{Aut}(T)^{k / m}$, and let $G=K \rtimes\left[\left(A \times S_{k / m}\right)\right.$ wr $\left.S_{m}\right]$ where $K$ acts regularly on itself and $\left(A \times S_{k / m}\right)$ wr $S_{m}$ acts naturally as a subgroup of $\operatorname{Aut}(K)=\operatorname{Aut}(T)$ wr $S_{k}$. Then $G$ is innately transitive with regular plinth $K$, and $\mathrm{C}_{G}(K)=C^{m}$ where $C$ is the straight diagonal subgroup of the left regular representation of $T^{k / m}$. Since $\mathrm{C}_{G}(K)$ is a subdirect product of $K$, we have that $G$ is of Diagonal Quotient type by [1, Remarks 10.2 and Proposition 10.3].

EXAMPLE 3 (Almost Simple Quotient type (ASQ)). Let $K$ be a nonabelian simple group and let $K_{0} / K_{1}$ be a section of $K$ where $K_{1}$ is corefree in $K_{0}$ and $K_{0}<K$. Let $\Omega=\left[K: K_{1}\right]$ and let $K$ act by right coset multiplication on $\Omega$. Let $G$ be the direct 
product $\left(K_{0} / K_{1}\right) \times K$ and define the action of $K_{0} / K_{1}$ on $\Omega$ by $\left(K_{1} u\right)^{K_{1} v}=K_{1} v^{-1} u$, for all $u \in K$ and $v \in K_{0}$. It turns out that $G$ is innately transitive on $\Omega$ of Almost Simple Quotient type.

EXAMPLE 4 (Product Action type (PA)). Now let $T$ be a nonabelian simple group, let $R$ be a proper subgroup of $T$, let $k>1$, let $A$ be a cyclic subgroup of $S_{k}$ of order $k$, let $S$ be a proper normal subgroup of the straight diagonal subgroup $\operatorname{Diag}\left(R^{k}\right)$ of $R^{k}$, and let $C=\operatorname{Diag}\left(R^{k}\right) / S$. Consider $G=C \times(T$ wr $A), G_{0}=S \times A<T$ wr $A$, and $\Omega=\left[G: G_{0}\right]$. If $S$ is nontrivial, then $G$ is innately transitive on $\Omega$ of Product Action type with plinth $K=T^{k}$. (Note: If $S=1$, then $G$ is innately transitive on $\Omega$ of Product Quotient type with plinth $K=T^{k}$.)

\section{Permutational transformations}

Let $G$ be a group acting on a set $\Omega$ and let $H$ be a group acting on a set $\Gamma$. Then $(\theta, \mu)$ is a permutational transformation from $G$ on $\Omega$ to $H$ on $\Gamma$ if $\theta: G \rightarrow H$ is a group homomorphism and $\mu: \Omega \rightarrow \Gamma$ is a function such that for all $g \in G$ and $\omega \in \Omega$, we have the intertwining relation $\left(\omega^{g}\right) \mu=(\omega) \mu^{(g) \theta}$. We say that $(\theta, \mu)$ is a permutational isomorphism if $\theta$ and $\mu$ are both bijections. Note that in the above definition, if $G$ acts faithfully on $\Omega$, it may not be true that $(G) \theta$ acts faithfully on $(\Omega) \mu$. The kernel of the action of $(G) \theta$ on $(\Omega) \mu$ is the image of $E=\left\{g \in G:\left(\omega^{g}\right) \mu=(\omega) \mu\right.$, for all $\left.\omega \in \Omega\right\}$ under $\theta$. We say that $(\theta, \mu)$ is a faithful permutational transformation if $E=\operatorname{ker} \theta$; that is, $(G) \theta$ acts faithfully on $(\Omega) \mu$. Recall that if $f$ is a function with domain $\Omega$, then a fibre of $f$ is a preimage of a point in the image of $f$. If $\omega \in \Omega$, then we denote the $f$-preimage of $(\omega) f$ by $[\omega]_{f}$.

Now if $\mathscr{B}$ is a $G$-invariant partition of $\Omega$, then there is an induced action of $G$ on $\mathscr{B}$. This is called a quotient action of $G$. We can reframe this concept in terms of permutational transformations. Let $E$ be the kernel of the action of $G$ on $\mathscr{B}$, and consider the faithful projective action of $G / E$ on $\mathscr{B}$. (If $G$ acts on a set $\Sigma$ and $N$ is a normal subgroup of $G$ contained in the kernel of the action of $G$ on $\Sigma$, then the projective action of $G / N$ on $\Sigma$ is defined by $\sigma^{N g}:=\sigma^{g}$ for all $\sigma \in \Sigma$ and $N g \in G / N$.) Let $\theta: G \rightarrow G / E$ be the canonical projection homomorphism and let $\mu: \Omega \rightarrow \mathscr{B}$ be the map which selects for each element of $\Omega$, the unique part of $\mathscr{B}$ it belongs to. Then $(\theta, \mu)$ is a faithful permutational transformation from the action of $G$ on $\Omega$ to the quotient action of $G$ on $\mathscr{B}$. Conversely, given a permutational transformation $(\theta, \mu)$ with domain $G$ acting on $\Omega$, the fibres of $\mu$ form a $G$-invariant partition $\mathscr{B}$ of $\Omega$, and it follows from the proposition below that the permutation group induced by the action of $G$ on $\mathscr{B}$ is permutationally isomorphic to the action of $(G) \theta$ on $(\Omega) \mu$. 
PROPOSITION 5.1 (The First 'Permutational' Isomorphism Theorem). Let $G$ be a group acting on a set $\Omega$ and let $H$ be a group acting on a set $\Gamma$, and suppose that $(\theta, \mu)$ is a permutational transformation from $G$ on $\Omega$ to $H$ on $\Gamma$. Then there is an action of $G / \operatorname{ker} \theta$ on the fibres of $\mu$ defined by $[\omega]_{\mu}^{(\operatorname{ker} \theta) g}:=\left[\omega^{g}\right]_{\mu}$, for all $g \in G$ and $\omega \in \Omega$, and this action is permutationally isomorphic to the action of $(G) \theta$ on $(\Omega) \mu$.

PROOF. Let $F_{\mu}$ be the set of fibres $\left\{[\omega]_{\mu}: \omega \in \Omega\right\}$ of $\mu$. The First Isomorphism Theorem in Group Theory states that the map $\Phi: G / \operatorname{ker} \theta \rightarrow(G) \theta$ defined by $((\operatorname{ker} \theta) g) \Phi=(g) \theta$ is a well-defined isomorphism. Similarly, the First Isomorphism Theorem for sets states that the map $\nu: F_{\mu} \rightarrow(\Omega) \mu$ defined by $\left([\omega]_{\mu}\right) \nu=(\omega) \mu$ is a well-defined bijection. So $\left(\Phi^{-1}, v^{-1}\right)$ and the intertwining relation, induce an action of $G / \operatorname{ker} \theta$ on $F_{\mu}$ as illustrated below. Let $g \in G$ and $\omega \in \Omega$. Then,

$$
[\omega]_{\mu}^{(\operatorname{ker} \theta)_{8}}=\left(((\omega) \mu) \nu^{-1}\right)^{((g) \theta) \Phi^{-1}}=\left((\omega) \mu^{(g) \theta}\right) \nu^{-1}=\left(\left(\omega^{8}\right) \mu\right) \nu^{-1}=\left[\omega^{g}\right]_{\mu},
$$

which is precisely the action given above. So we see that $(\Phi, \nu)$ is a permutational isomorphism from the action of $G / \operatorname{ker} \theta$ on $F_{\mu}$ (given above) to the action of $(G) \theta$ on $(\Omega) \mu$.

The following lemma lists some basic results on faithful permutational transformations.

LEMMA 5.2. Let $G$ be a group acting on a set $\Omega$ and let $H$ be a group acting on a set $\Gamma$, and suppose that $(\theta, \mu)$ is a faithful permutational transformation from $G$ on $\Omega$ to $H$ on $\Gamma$. Then we have the following:

(1) If $G$ is transitive on $\Omega$, then $(G) \theta$ is transitive on $(\Omega) \mu$.

(2) If $(G) \theta$ is semiregular, then $G / \operatorname{ker} \theta$ is semiregular on $\Omega$ (in its projective action).

(3) If $G$ is faithful and innately transitive on $\Omega$ and $\theta$ is nontrivial, then $(G) \theta$ is innately transitive on $(\Omega) \mu$.

(4) If $G$ is faithful and primitive on $\Omega$ and $\theta$ is nontrivial, then $(G) \theta^{(\Omega) \mu}$ is permutationally isomorphic to $G^{\Omega}$.

Proof. (1) Suppose $G$ is transitive and let $\omega_{1}, \omega_{2} \in \Omega$. Since $G$ is transitive, there exists $g \in G$ such that $\omega_{1}^{g}=\omega_{2}$. Hence $\left(\omega_{1}\right) \mu^{(g) \theta}=\left(\omega_{1}^{g}\right) \mu=\left(\omega_{2}\right) \mu$ and $(G) \theta$ is transitive on $(\Omega) \mu$.

(2) Suppose $(G) \theta$ is semiregular and suppose $\omega^{(\text {ker } \theta) g}=\omega$ for some $g \in G$ and $\omega \in \Omega$. Then $\omega^{g}=\omega$ and hence $\left(\omega^{g}\right) \mu=(\omega) \mu$. So $(\omega) \mu^{(g) \theta}=(\omega) \mu$ and therefore $(g) \theta=1$. Thus $g \in \operatorname{ker} \theta$ and $G / \operatorname{ker} \theta$ is semiregular on $\Omega$.

(3) Suppose $G$ is innately transitive on $\Omega$ with plinth $K$. Assume that $(K) \theta=1$ and fix a point $\omega \in \Omega$. Since $K$ is transitive, every element of $\Omega$ is of the form $\omega^{y}$ for some 
$y \in K$. Hence every element of $(\Omega) \mu$ is of the form $\left(\omega^{y}\right) \mu=(\omega) \mu^{(y) \theta}=(\omega) \mu$ and thus $(\Omega) \mu$ has only one element. This implies that $(G) \theta$ is the trivial group (as it acts faithfully on $(\Omega) \mu$, which is a contradiction. Therefore $(K) \theta$ is a nontrivial normal subgroup of $(G) \theta$ (as $K$ is a normal subgroup of $G$ ). If $M$ is a normal subgroup of $(G) \theta$ contained in $(K) \theta$, then the preimage of $M$ under $\theta$ is a normal subgroup of $G$ contained in $K$. It then follows that $(K) \theta$ is a minimal normal subgroup of $(G) \theta$. By $(1),(K) \theta$ is transitive on $(\Omega) \mu$ and hence $(G) \theta$ is innately transitive on $(\Omega) \mu$ with plinth $(K) \theta$.

(4) Suppose $G^{\Omega}$ is primitive. Then the fibres of $\mu$ are trivial as they form a $G$-invariant partition of $\Omega$ and $(\theta, \mu)$ is faithful. Since $\theta$ is nontrivial, it follows that $[\omega]_{\mu}=\{\omega\}$ for all $\omega \in \Omega$. Hence $\mu$ is injective and thus $\operatorname{ker} \theta=1$ (as $(\theta, \mu)$ is faithful). By Theorem 5.1, we have that $G^{\Omega}$ is permutationally isomorphic to $(G) \theta^{(\Omega) \mu}$.

Similarly, one can prove that 2-transitivity and quasiprimitivity are preserved by permutational transformations and we leave this as an exercise for the reader. Now suppose a group $G$ acts transitively on a set $\Omega$, and $(\theta, \mu)$ is a faithful permutational transformation from $G$ on $\Omega$ to the action of a group $H$ on a set $\Delta$. Recall that if $K$ is a transitive normal subgroup of $G$ and $\alpha \in \Omega$, then $G=K G_{\alpha}$. Suppose that we have another homomorphism $\theta^{\prime}: G \rightarrow H$ such that $\left(\theta^{\prime}, \mu\right)$ is a permutational transformation where the restriction of $\theta^{\prime}$ to $K$ is equal to the restriction of $\theta$ to $K$. Then for all $y \in K$ and $g \in G_{\alpha}$, we have

$$
\begin{aligned}
(g) \theta^{\prime}\left(g^{-1}\right) \theta(y) \theta & =(g) \theta^{\prime}\left(g^{-1} y g\right) \theta\left(g^{-1}\right) \theta=(g) \theta^{\prime}\left(g^{-1} y g\right) \theta^{\prime}\left(g^{-1}\right) \theta \\
& =(y) \theta^{\prime}(g) \theta^{\prime}\left(g^{-1}\right) \theta=(y) \theta(g) \theta^{\prime}\left(g^{-1}\right) \theta .
\end{aligned}
$$

Therefore $(g) \theta^{\prime}\left(g^{-1}\right) \theta \in \mathrm{C}_{(G) \theta}((K) \theta) \cap\left(G_{\alpha}\right) \theta$. Note that for all $h \in G_{\alpha}$, we have $(\alpha) \mu^{(h) \theta}=\left(\alpha^{h}\right) \mu=(\alpha) \mu$ and hence every element of $\left(G_{\alpha}\right) \theta$ fixes $(\alpha) \mu$. Similarly $\left(G_{\alpha}\right) \theta^{\prime}$ fixes $(\alpha) \mu$. By Lemma 5.2, $(K) \theta$ is innately transitive on $(\Omega) \mu$ and hence $\mathrm{C}_{(G) \theta}((K) \theta)$ is semiregular (by Lemma $1.1(5)$ ). So it follows that $\mathrm{C}_{(G) \theta}((K) \theta) \cap$ $\left(G_{\alpha}\right) \theta=1$ and $(g) \theta=(g) \theta^{\prime}$. Therefore $\theta=\theta^{\prime}$ as $G=K G_{\alpha}$. Hence every faithful permutational transformation with domain $G$ acting on $\Omega$, is determined by its action on a transitive normal subgroup $K$. So we see that the faithful permutational transformations whose domain $G$ is innately transitive, are completely determined by the plinth of $G$.

\section{Generalising Praeger and Shalev's results}

In Praeger and Shalev's paper [10], they extend classical results of primitive groups to quasiprimitive groups. These results include bounds on the order of a quasiprimitive group in terms of its degree, a bound on the number of fixed points of a quasiprimitive 
group containing special permutations, a bound on the base size of a quasiprimitive group, and a bound on the minimal degree of a quasiprimitive group. Praeger and Shalev also give a nice exposition of the literature in [10], which we do not repeat here.

Below we generalise the following results of Praeger and Shalev: Theorems 2.1, $2.2,4.1,4.2,4.4,4.3,7.2$.

THEOREM 6.1. Let $G$ be an innately transitive permutation group on a set $\Omega$ with plinth $K$, and let $n=|\Omega|$. Then

(1) if $G$ contains a $p$-cycle, for some prime $p$, then $G$ is primitive;

(2) if for some prime $p$, there is an element in $G$ of order $p$ with $q$ cycles of length $p$ in $\Omega$, where $2 \leq q<p$. Then either,

(i) $n-q p \leq 5 q / 2-2$, or

(ii) $G$ is quasiprimitive;

(3) either $|G| \leq n ! /((n+1) / 2)$ ! or $G$ contains $A_{n}$;

(4) either $|G|<4^{n}$ or $G$ contains $A_{n}$;

(5) there exist constants $d$ and $d^{\prime}$ such that either

(i) $|G| \leq n^{d \log (n)}$, or

(ii) for positive integers $m, k, l$ such that $k \leq d^{\prime}, l \leq d^{\prime}$ and $m>4 d^{\prime}$, we have $G / C_{G}(K) \leq S_{m}$ wr $S_{l}$ with $K \cong A_{m}^{l}$ and $\left(A_{m-k}\right)^{l} \leq K_{\alpha} \leq\left(S_{m-k} \times S_{k}\right)^{l} \cap K$ where $\alpha$ is a point in $\Omega$. Also, $\left|\mathrm{C}_{G}(K)\right| \leq(k !)^{l} \leq\left(d^{\prime} !\right)^{d^{\prime}}$;

(6) either $b(G) \leq 4 \sqrt{n} \log (n)$ or $G$ contains $A_{n}$;

(7) either $m(G) \geq(\sqrt{n}-1) / 2$ or $G$ contains $A_{n}$.

REMARKS. The bound in (3), which was originally found by Bochert [2] for primitive groups, is reasonably accurate for small degrees and was the best bound of its time (circa 1889). The bound in (4), found by Praeger and Saxl [9] for primitive groups, has the advantage that it has a simple algebraic expression and holds for all innately transitive groups. Cameron's bound, which is generalised as (5) above, is asymptotically the best of the bounds above on the orders of primitive groups. However, its proof depends on the Classification of Finite Simple Groups.

\section{Preliminary results}

In order to prove Theorem 6.1, we will need some basic facts first. The lemma below generalises (2) and (5) of Lemma 1.1, and to some extent, (4) as well.

LEMMA 7.1. Let $G$ be a finite innately transitive permutation group acting on a set $\Omega$ with plinth $K$, let $H$ be a group acting on a set $\Gamma$, and suppose that $(\theta, \mu)$ is 
a faithful permutational transformation from $G$ on $\Omega$ to $H$ on $\Gamma$. Then the action of $(G) \theta$ on $(\Omega) \mu$ is permutationally isomorphic to the action of $G / \operatorname{ker} \theta$ on the orbits of $\operatorname{ker} \theta$ in $\Omega$, defined by $\left(\omega^{\mathrm{ker} \theta}\right)^{(\mathrm{ker} \theta) g}=\left(\omega^{g}\right)^{\mathrm{ker} \theta}$ for all $g \in G$ and $\omega \in \Omega$. Moreover, $\operatorname{ker} \theta \leq \mathrm{C}_{G}(K)$ and if $\Delta$ is the $\operatorname{ker} \theta$-orbit of a point $\alpha$ in $\Omega$, then $K_{\alpha}$ is a normal subgroup of $K_{\Delta}$ and $\operatorname{ker} \theta \cong K_{\Delta} / K_{\alpha}$.

PROOF. Let $F_{\mu}$ be the set of fibres of $\mu$ and let $\Sigma$ be the set of $\operatorname{ker} \theta$-orbits in $\Omega$. Then the map $\nu: F_{\mu} \rightarrow \Sigma$ defined by $\left([\omega]_{\mu}\right) \nu=\omega^{\operatorname{ker} \theta}$ is a bijection and it induces a natural action of $G / \operatorname{ker} \theta$ on $F_{\mu}$ which is permutationally isomorphic to the action of $G / \operatorname{ker} \theta$ on $\Sigma$ given above. Therefore, by the First Permutational Isomorphism Theorem, the action of $(G) \theta$ on $(\Omega) \mu$ is permutationally isomorphic to the action of $G / \operatorname{ker} \theta$ on the orbits of $\operatorname{ker} \theta$.

Since $K$ is transitive, we have that $(K) \theta$ is transitive by Lemma 5.2. So $K$ is not contained in $\operatorname{ker} \theta$ (as $(K) \theta$ is nontrivial) and hence by the minimality of $K$, we see that $\operatorname{ker} \theta$ is a normal subgroup of $G$ that intersects $K$ trivially. It then follows that $\operatorname{ker} \theta$ centralises $K$.

Let $\alpha$ be a point in $\Omega$ and let $\Delta=\alpha^{\text {ker } \theta}$. Let $y \in K_{\Delta}$ and suppose there are elements $c, d$ of $\operatorname{ker} \theta$ such that $\alpha^{y}=\alpha^{c}$ and $\alpha^{y}=\alpha^{d}$. Since $\operatorname{ker} \theta$ is a subgroup of $C_{G}(K)$, by Lemma 1.1(5), $\operatorname{ker} \theta$ is semiregular and hence $G_{\alpha} \cap \operatorname{ker} \theta=1$. Now $c d^{-1} \in G_{\alpha} \cap \operatorname{ker} \theta$ and hence $c=d$. So there is a map $\varphi: K_{\Delta} \rightarrow \operatorname{ker} \theta$ where for each element $y \in K_{\Delta}$, $(y) \varphi$ is the unique element of $\operatorname{ker} \theta$ such that $y(y) \varphi \in G_{\alpha}$. We prove now that $\varphi$ is a group homomorphism. Let $y_{1}, y_{2} \in \operatorname{ker} \theta$. Then $\alpha^{y_{1} y_{2}\left(y_{1}\right) \varphi\left(y_{2}\right) \varphi}=\left(\alpha^{y_{1}\left(y_{1}\right) \varphi}\right)^{y_{2}\left(y_{2}\right) \varphi}$ as $\operatorname{ker} \theta$ centralises $K$. Therefore $\alpha^{y_{1} y_{2}\left(y_{1}\right) \varphi\left(y_{2}\right) \varphi}=\alpha$ and so by uniqueness, $\left(y_{1}\right) \varphi\left(y_{2}\right) \varphi=$ $\left(y_{1} y_{2}\right) \varphi$ and $\varphi$ is a homomorphism. Clearly $\operatorname{ker} \varphi=K_{\alpha}$. Therefore, $K_{\alpha}$ is a normal subgroup of $K_{\Delta}$ and $\operatorname{ker} \theta \cong K_{\Delta} / K_{\alpha}$.

We will frequently make use of the following lemma in the proof of Theorem 6.1.

LEMMA 7.2. Let $G$ be an innately transitive permutation group on a set $\Omega$ with plinth $K$, let $H$ be a group acting on a set $\Gamma$, let $(\theta, \mu)$ be a faithful permutational transformation from $G^{\Omega}$ to $H^{\Gamma}$, and let $m=|(\Omega) \mu|>1$. If $(G) \theta$ contains $A_{m}$, then one of the following holds:

(1) $G$ contains $A_{m}$.

(2) $G$ is of $A S Q_{\text {reg }}$ type, $G=A_{m-1} \times A_{m}$ or $G=\left(A_{m-1} \times A_{m}\right) .2$, and $K=A_{m}$.

(3) $G$ has degree 15, $G$ is of Almost Simple Quotient type, $K=A_{5}, \operatorname{ker} \theta$ is cyclic of order 3 , and $|G:(\operatorname{ker} \theta \times K)|=1,2$.

ProOF. First, if $G^{\Omega}$ is primitive, then by Lemma $5.2,(G) \theta^{(\Omega) \mu}$ is permutationally isomorphic to $G^{\Omega}$ and (1) holds. This is also true in the general case when $\operatorname{ker} \theta=1$ (by Theorem 5.1). Suppose $\operatorname{ker} \theta>1$ (so necessarily, $G$ is imprimitive). If $\operatorname{ker} \theta$ is transitive, then $\mathrm{C}_{G}(K)$ is transitive, as $\operatorname{ker} \theta \leq \mathrm{C}_{G}(K)$ by Lemma 7.1, and hence $G$ 
is primitive (see [1, Lemma 5.1])-a contradiction. So $\operatorname{ker} \theta$ is an intransitive normal subgroup of $G$. Therefore $G^{\Omega}$ is not quasiprimitive and hence $K$ is nonabelian. Let $\alpha \in \Omega$ and let $\Delta=\alpha^{\text {ker } \theta}$. Then by Lemma 7.1, $K_{\alpha}$ is a proper normal subgroup of $K_{\Delta}$ and $\operatorname{ker} \theta \cong K_{\Delta} / K_{\alpha}$. Let $c=|\operatorname{ker} \theta|$ and let $n=|\Omega|=m c$.

Since $K$ is nonabelian, we must have that $m \geq 5$. So $A_{m}$ is a simple group and hence $K \cong(K) \theta=A_{m}$ as $A_{m}$ is the unique minimal normal subgroup of both $S_{m}$ and itself. The $m$ fibres of $\mu$ are simple the orbits of $\operatorname{ker} \theta$ in $\Omega$. So $K_{\Delta}=A_{m-1}$ and $K_{\alpha}$ is normal in $K_{\Delta}$. We have two subcases: $m=5$ or $m \neq 5$. In the latter case, $K_{\Delta}$ is simple, and since $K_{\alpha}$ is a proper normal subgroup of $K_{\Delta}$, we have that $K_{\alpha}=1$. Thus $\operatorname{ker} \theta \cong K_{\Delta}=A_{m-1}$ and $G$ is of $A S Q_{\text {reg }}$ type. Note that the only subgroups of $S_{m}$ that intersect $A_{m}$ in $A_{m-1}$ are $S_{m-1}$ and $A_{m-1}$. Since $A_{m} \leq(G) \theta \cong G / \operatorname{ker} \theta \leq S_{m}$ and $G \geq \operatorname{ker} \theta \times K=A_{m-1} \times A_{m}$, it follows that $G \in\left\{A_{m-1} \times A_{m},\left(A_{m-1} \times A_{m}\right) .2\right\}$, and we are in case (2).

Finally, let $m=5$. Then $K_{\Delta}=A_{4}$ and $K_{\alpha} \in\left\{1, V_{4}\right\}$ as $K_{\alpha}$ is a proper normal subgroup of $K_{\Delta}$. If $K_{\alpha}=1$, then $G$ is of Almost Simple Quotient type with a regular plinth $K, \operatorname{ker} \theta \cong K_{\Delta} / K_{\alpha} \cong A_{4}$, and by a similar argument as before, $G \in\left\{A_{m-1} \times A_{m},\left(A_{m-1} \times A_{m}\right) .2\right\}$ where $m=5$, and we are in case (2). So suppose that $K_{\alpha}=A_{4}$. Then $\operatorname{ker} \theta \cong K_{\Delta} / K_{\alpha} \cong \mathbb{Z}_{3}$ and $n=5\left|K_{\Delta}: K_{\alpha}\right|=15$. Since ( $G) \theta \cong G / \operatorname{ker} \theta \leq S_{5}$ and $G \geq \operatorname{ker} \theta \times K=\mathbb{Z}_{3} \times A_{5}$, case (3) holds.

Notice that for the case where the permutational transformation $(\theta, \mu)$ is just the quotient action of $G$ on $\mathscr{C}$, the orbits of $\mathrm{C}_{G}(K)$, we have that if $c=|\mathscr{C}|$ and $G^{\mathscr{C}}$ contains $A_{c}$, then Lemma 7.2 implies that one of the following holds:

(1) $G$ contains $A_{c}$;

(2) $G$ is of $A S Q_{\text {reg }}$ type, $G=A_{c-1} \times A_{c}$ or $G=\left(A_{c-1} \times A_{c}\right) .2$, and $K=A_{c}$;

(3) $G$ has degree 15.

The only innately transitive groups of degree 15 that are not quasiprimitive, are isomorphic to one of the two groups $\mathbb{Z}_{3} \times A_{5}$ or $\left(\mathbb{Z}_{3} \times A_{5}\right) .2$. One can construct the latter group $G$ as follows. Note that $\operatorname{Aut}\left(\mathbb{Z}_{3} \times A_{5}\right) \cong\left\langle a: a^{2}=1\right\rangle \times S_{5}$ and let $c=(a,(1,2)) \in \operatorname{Aut}\left(\mathbb{Z}_{3} \times A_{5}\right)$. It turns out that $G=\left(\mathbb{Z}_{3} \times A_{5}\right) \times\langle c\rangle$. The point stabiliser for this group is $H \rtimes\langle c\rangle \cong S_{4}$, where $H \cong A_{4}$.

Praeger and Shalev showed that if $G$ is a quasiprimitive group on a set $\Omega$, and $\mathscr{B}$ is a $G$-invariant partition for $G$ in $\Omega$, then $b_{\Omega}(G) \leq b_{\mathscr{B}}(G)$. They used the fact that $G$ acts faithfully on $\mathscr{B}$, which does not necessarily hold for an arbitrary innately transitive group. Similarly, they showed that the the minimal base size of $G$ on $\Omega$ is no less than $s \cdot m_{\mathscr{B}}(G)$ where $s$ is the size of a block in $\mathscr{B}$. A more general result can be achieved in the context of faithful permutational transformations.

LEMMA 7.3. Let $G$ be an innately transitive permutation group on a set $\Omega$ with plinth $K$, let $H$ be a finite transitive permutation group on a set $\Gamma$, let $(\theta, \mu)$ be a 
faithful permutational transformation from $G$ on $\Omega$ to $H$ on $\Gamma$, and let $c=|\Omega| /|(\Omega) \mu|$. Then

(1) $b_{\Omega}(G) \leq b_{(\Omega) \mu}((G) \theta)$;

(2) $m_{\Omega}(G) \geq c \cdot m_{(\Omega) \mu}((G) \theta)$.

PROOF. (1) Let $\Delta_{1}, \ldots, \Delta_{b}$ be a base for $(G) \theta$ in $(\Omega) \mu$, and let $\alpha_{1}, \ldots, \alpha_{b}$ be elements of $\Omega$ such that $\left(\alpha_{i}\right) \mu \in \Delta_{i}$ for all $i$. Suppose $g \in G$ fixes each of the $\alpha_{i}$. Hence $(g) \theta$ fixes the $\Delta_{i}$, and therefore $g \in \operatorname{ker} \theta$ as $\Delta_{1}, \ldots, \Delta_{b}$ is a base. But $\operatorname{ker} \theta$ is semiregular on $\Omega$ and hence $g=1$ and $\alpha_{1}, \ldots, \alpha_{b}$ is a base for $G$ in $\Omega$. Therefore $b_{\Omega}(G) \leq b_{(\Omega) \mu}((G) \theta)$.

(2) First note that the $\operatorname{ker} \theta$ orbits have a common cardinality (as $G$ is transitive) which is equal to the constant $c$ (by Lemma 7.1). Let $g$ be a non-identity element of $G$ and set $m=m_{(\Omega) \mu}((G) \theta)$. If $g \notin \operatorname{ker} \theta$, then $(g) \theta$ acts nontrivially on $(\Omega) \mu$ and hence moves at least $\mathrm{cm}$ points of $\Omega$. If $g \in \operatorname{ker} \theta$, then since $\operatorname{ker} \theta$ is semiregular, $g$ must move every point in $\Omega$. Therefore $m_{\Omega}(G) \geq c \cdot m$.

The following result will be used to settle a case in the proof of Theorem 6.1(7).

LEMMA 7.4. Let $n \geq 5$ and consider the natural action of Aut $\left(A_{n}\right)$ on $A_{n}$. Consider $\operatorname{Hol}\left(A_{n}\right)=A_{n} \rtimes \operatorname{Aut}\left(A_{n}\right)$ in its natural action on $A_{n}$. Then

$$
m\left(\operatorname{Hol}\left(A_{n}\right)\right)=m\left(\operatorname{Aut}\left(A_{n}\right)\right)=n ! / 2-(n-2) ! .
$$

PROOF. First we assume that $n \neq 6$ and identify the action of $\operatorname{Aut}\left(A_{n}\right)$ with the conjugation action of $S_{n}$ on $A_{n}$. Since $\operatorname{Aut}\left(A_{n}\right) \leq \operatorname{Hol}\left(A_{n}\right)$, it is clear that $m\left(\operatorname{Hol}\left(A_{n}\right)\right) \leq m\left(\operatorname{Aut}\left(A_{n}\right)\right)$. Let $g \in \operatorname{Hol}\left(A_{n}\right)$ such that $g \neq 1$ and $g$ permutes $m\left(\operatorname{Hol}\left(A_{n}\right)\right)$ points. Since $\operatorname{Hol}\left(A_{n}\right)$ is transitive, we may assume that $g$ fixes the identity of $A_{n}$ and hence $g \in \operatorname{Aut}\left(A_{n}\right)$. So $m\left(\operatorname{Hol}\left(A_{n}\right)\right) \geq m\left(\operatorname{Aut}\left(A_{n}\right)\right)$ and therefore $m\left(\operatorname{Hol}\left(A_{n}\right)\right)=m\left(\operatorname{Aut}\left(A_{n}\right)\right)$. By the definition of the action, $g$ fixes exactly $\left|\mathrm{C}_{A_{n}}(g)\right|$ points and so $m\left(\operatorname{Hol}\left(A_{n}\right)\right)=\left|A_{n}\right|-\left|\mathrm{C}_{A_{n}}(g)\right|$. By the definition of minimal degree, $\left|\mathrm{C}_{A_{n}}(g)\right|$ is the maximum number of fixed points over all $g \in \operatorname{Aut}\left(A_{n}\right)$ with $g \neq 1$. Let $\tau$ be the automorphism induced by the transposition $(n-1, n)$. Since $(n-1, n)$ centralises itself and $A_{n-2}$, we have that $\tau$ has $(n-2)$ ! fixed points. So $m\left(\operatorname{Aut}\left(A_{n}\right)\right) \leq$ $n ! / 2-(n-2)$ !. It is clear that the transpositions of $S_{n}$ centralise the most number of elements of $A_{n}$. Therefore $m\left(\operatorname{Aut}\left(A_{n}\right)\right)=n ! / 2-(n-2)$ !.

It can be calculated using a computer program such as GAP, that $(*)$ holds for $n=6$. Therefore $(*)$ holds for all $n \geq 5$.

\section{Proof of Theorem 6.1}

We can finally prove Theorem 6.1 . 
PROOF. We prove the parts of Theorem 6.1 consecutively. Let $K$ be the plinth of $G$, let $c=\left|\mathrm{C}_{G}(K)\right|$, and let $\mathscr{C}$ be the set of $\mathrm{C}_{G}(K)$-orbits in $\Omega$.

(1) Let $g \in G$ be a $p$-cycle of $\Omega$. Let $\alpha_{1}, \alpha_{2}, \ldots, \alpha_{p} \in \Omega$ be such that $\alpha_{1}^{g}=$ $\alpha_{2}, \ldots, \alpha_{p-1}^{g}=\alpha_{p}$ and $\alpha_{p}^{g}=\alpha_{1}$. For all $i=1, \ldots p$, let $\Delta_{i}=\alpha_{i}^{\mathrm{C}_{G}(K)}$. Now if $|\Omega|=p$, then $G$ is clearly primitive (since the size of a block for $G$ divides the size of $\Omega$ ). Suppose $|\Omega|>p$. Since $\mathrm{C}_{G}(K)$ is semiregular, $g \notin \mathrm{C}_{G}(K)$ and hence $G \neq \mathrm{C}_{G}(K)$. By Lemma $1.1(4), K$ acts faithfully on $\mathscr{C}$ and hence $G$ acts nontrivially on $\mathscr{C}$. Also, $\mathrm{C}_{G}(K)$ is the kernel of the action of $G$ on $\mathscr{C}$ (by Lemma 1.1 (4)) and hence $g$ induces a $p$-cycle $\Delta_{1}, \ldots, \Delta_{p}$ on $\mathscr{C}$.

Now the points in the union of the $\Delta_{i}$ must consist wholly of elements of the $p$-cycle, $\alpha_{1}, \ldots, \alpha_{p}$, otherwise $g$ would fix a point in one of the $\Delta_{i}$ and hence fix an orbit in its action on $\mathscr{C}$ (and the $\Delta_{i}$ would not be pairwise distinct). So the size of the orbits must divide $p$. Since the $\Delta_{i}$ are distinct, the orbits in $\mathscr{C}$ must be singleton sets, and hence $\mathrm{C}_{G}(K)=1$. Therefore $G$ is quasiprimitive by Lemma $1.1(1)$ and so $G$ is primitive by the proof of Praeger and Shalev's result [10, Theorem 2.1].

(2) Suppose that $G$ is not quasiprimitive, so by Lemma 1.1 (1), $1<c<n$. Let $g \in G$ be an element of order $p$ with $q$ cycles of length $p$ in $\Omega$ (where $2 \leq q<p$ ), and let $f$ be the number of fixed points of $g$ in $\Omega$, that is, $f=n-q p$. If $f=0$, that is $n=q p$, then (i) holds. Suppose $f>0$. Then $g \notin \mathrm{C}_{G}(K)$ since $\mathrm{C}_{G}(K)$ is semiregular and $g$ fixes at least one point. So by Lemma 1.1 (4), $G$ acts nontrivially on $\mathscr{C}$ with kernel $\mathrm{C}_{G}(K)$ and hence $g \neq 1$ and each cycle of $g$ of length $p$ in $\mathscr{C}$ corresponds to $c$ cycles of length $p$ of $g$ in $\Omega$. Since $q<p$, it follows that $g$ has $q^{\prime}=q / c<p$ cycles of length $p$ in $\mathscr{C}$, and $f^{\prime}=f / c$ fixed points in $\mathscr{C}$. Now $G^{\mathscr{C}}$ acts quasiprimitively on $\mathscr{C}$, and so by Praeger and Shalev's result, one of the following holds:

(a) $f^{\prime} \leq 5 q^{\prime} / 2-2$,

(b) $G^{\mathscr{C}}=A_{n / c}$ or $S_{n / c}$,

(c) $G^{\mathscr{C}}=A_{d}$ or $S_{d}$ on ordered pairs $\left(n / c=\left(\begin{array}{l}d \\ 2\end{array}\right), d \geq p, q^{\prime}=d-(p+1) / 2\right)$.

In the first case, $f / c \leq 5 q / 2 c-2$ and hence $f \leq 5 q / 2-2 c \leq 5 q / 2-2$, so (i) holds. Suppose now that (b) holds. By Lemma 7.2 either $G$ is of Almost Simple Quotient type and $A_{m-1} \times A_{m} \leq G \leq\left(A_{m-1} \times A_{m}\right) .2$ where $m=n / c>5$, or $n=15$ and $c=3$. In the latter case, computer calculations (in GAP) show that $(p, q) \in$ $\{(3,5),(3,7),(5,3)\}$ and hence (i) holds. In the former case, $p>c=\left|A_{m-1}\right|$, and since $m \geq p$ we have $p>(p-1) ! / 2$ which is impossible for $p \geq 5$. Hence $p=3$, but in this case $m \geq 6$ and again this is not true.

Finally, consider case (c). Here $q^{\prime}=d-(p+1) / 2 \geq(p-1) / 2$ and as $q=c q^{\prime}<p$, it follows that $c=2, q=p-1$, and $d=p$. In this case $g$ has no fixed points on $\mathscr{C}$, and hence no fixed points on $\Omega$, and (i) holds.

(3) If $G$ contains a 3-cycle, then by Theorem 6.1 (1), $G$ is primitive and the conclusion follows from Bochert's result. If $G$ does not contain a 3-cycle, then by Bochert's 
original argument (see [2] or [3, Theorem 3.3B]), we have that $|G| \leq n ! /((n+1) / 2)$ !. (The author would like to thank Dr. Peter M. Neumann for suggesting the proof of this result.)

(4) Recall that $G^{\mathscr{C}}$ acts faithfully and quasiprimitively on $\mathscr{C}$, and so by Praeger and Shalev's result [10, Theorem 4.2], if $G^{\mathscr{C}}$ does not contain $A_{n / c}$, then $|G|=c\left|G^{\mathscr{C}}\right|<$ $c 4^{n / c}$. Now if $c 4^{m / c} \leq 4^{m}$ for some $m \geq c$, then $c 4^{(m+1) / c}=c 4^{m / c} 4^{1 / c} \leq 4^{m} 4^{1 / c} \leq 4^{m+1}$. The smallest case, $m=c$, holds since $c 4^{1}=4 c \leq 4^{c}$. So by induction, we see that $c 4^{m / c} \leq 4^{m}$ for all $m \geq c$. Therefore $|G|<4^{n}$.

So assume now that $A_{n / c} \subseteq G^{\mathscr{C}}$. By Lemma 7.2, one of the following holds: $G$ is quasiprimitive, $G$ is isomorphic to a subgroup of $\left(A_{n / c-1} \times A_{n / c}\right) \cdot 2$, or $n=15$ and $c=3$. In the first case, the result holds by [10, Theorem 4.2]. In the latter case, $|G|=c\left|G^{\mathscr{C}}\right| \leq 3 \cdot 5 !=360<4^{15}$. In the case that $G$ is embedded in $\left(A_{n / c-1} \times A_{n / c}\right) .2$, recall that $n=(n / c) ! / 2$ since the plinth $A_{n / c}$ is regular, and hence $|G| \leq n^{2} / 2$. Now $n \mapsto 4^{n}-n^{2}$ is an increasing function on the natural numbers, and is equal to 3 when $n=1$. So $4^{n}-n^{2}>0$ for all positive integers $n$ and hence $|G|<4^{n}$.

(5) Since $G^{\mathscr{C}}$ is quasiprimitive on $\mathscr{C}$, by Praeger and Shalev's result [10, Theorem 4.4] (which depends on Cameron's result and hence the Classification of Finite Simple Groups), there exist constants $d$ and $d^{\prime}$ such that either $\left|G^{\mathscr{C}}\right| \leq(n / c)^{d \log (n / c)}$ or, for positive integers $m, k, l$ such that $k \leq d^{\prime}, l \leq d^{\prime}$ and $m>4 d^{\prime}$, we have $G^{\mathscr{C}} \leq S_{m}$ wr $S_{l}$ with $K^{\mathscr{C}}=A_{m}^{l}$ and $\left(A_{m-k}\right)^{l} \leq K_{\Delta}^{\mathscr{C}} \leq\left(S_{m-k} \times S_{k}\right)^{l} \cap K$ (for some $\Delta \in \mathscr{C}$ ). In the former case, $|G|=c\left|G^{\mathscr{C}}\right| \leq c(n / c)^{d \log (n / c)} \leq n^{d \log (n)}$, and we have that (i) holds.

So assume that we are in the second case and let $J=\left(A_{m-k}\right)^{l}<K$ (note $K^{\mathscr{C}} \cong K$ ). Since $J$ is a minimal normal subgroup of $K_{\Delta}$, we have two cases; namely $J \cap K_{\alpha}=1$ or $J \leq K_{\alpha}$. In the latter case, $\left|C_{G}(K)\right|=\left|K_{\Delta}\right| /\left|K_{\alpha}\right|$ (by Lemma 1.1 (1)) and $K_{\Delta} \leq \mathrm{N}_{K}\left(K_{\alpha}\right) \leq \mathrm{N}_{K}\left(A_{m-k}^{l}\right)=\left(S_{m-k} \times S_{k}\right)^{l} \cap K$ and hence $\left|\mathrm{C}_{G}(K)\right| \leq(k !)^{l} \leq\left(d^{\prime} !\right)^{d^{\prime}}$. So (ii) holds. So suppose that $J \cap K_{\alpha}=1$. Then $|J|$ divides $\left|K_{\Delta}: K_{\alpha}\right|$ and hence $((m-k) ! / 2)^{l}$ divides $c$. Now

$$
\begin{aligned}
|G| & =c\left|G^{\mathscr{C}}\right| \leq c(m !)^{l} l !=\frac{n(m !)^{l} l !}{\left(\begin{array}{l}
m \\
k
\end{array}\right)^{l}}=n(l !)(k !(m-k) !)^{l} \\
& \leq n(l !)(k !)^{l} 2^{l} c \leq n c\left(d^{\prime} !\right)\left(d^{\prime} !\right)^{d^{\prime}} 2^{d^{\prime}}=n c 2^{d^{\prime}}\left(d^{\prime} !\right)^{d^{\prime}+1} \leq n^{2} b\left(d^{\prime}\right)
\end{aligned}
$$

where $b\left(d^{\prime}\right)=2^{d^{\prime}}\left(d^{\prime} !\right)^{d^{\prime}+1}$. (We could replace the constant $d^{\prime}$, given by [10, Theorem 4.4], with $b\left(d^{\prime}\right)$.) So we have that $G$ satisfies (i).

(6) If $A_{n / c} \nsubseteq G^{\mathscr{C}}$, then by Lemma 7.3,

$$
b(G)=b_{\Omega}(G) \leq b_{\mathscr{C}}(G)=4(n / c)^{1 / 2} \log (n / c) \leq 4 \sqrt{n} \log (n) .
$$

On the other hand, if $A_{n / c} \subseteq G^{\mathscr{C}}$, then by Lemma 7.2 one of the following holds: $G$ is quasiprimitive, $G \leq \operatorname{Hol}\left(A_{m}\right)$ for some $m \geq 5$, or $n=15$. In the third case, $b(G) \leq$ 
$15 \leq 4 \sqrt{15} \log (15)$. If $G \leq \operatorname{Hol}\left(A_{m}\right)($ where $m \geq 5)$, then $b(G) \leq b\left(\operatorname{Hol}\left(A_{m}\right)\right)$. $\operatorname{Now} \operatorname{Hol}\left(A_{m}\right)$ is primitive (as the point stabilizer of 1 in $\operatorname{Hol}\left(A_{m}\right)$ is $\operatorname{Aut}\left(A_{m}\right)$, which is maximal in $\left.\operatorname{Hol}\left(A_{m}\right)\right)$, and so it follows from Babai's result that $b(G) \leq 4 \sqrt{n} \log (n)$. If $G$ is quasiprimitive, then by Praeger and Shalev's result [10, Lemma 5.1], either $b(G) \leq 4 \sqrt{n} \log (n)$ or $G$ contains $A_{n}$.

(7) Suppose $G^{\mathscr{C}}$ does not equal $A_{n / c}$ or $S_{n / c}$. Then by Theorem [10, Theorem 7.2] and Lemma 7.3, $m_{\Omega} \geq c \cdot m_{\mathscr{C}}(G) \geq c(\sqrt{n / c}-1) / 2 \geq(\sqrt{n}-1) / 2$. Suppose now that $G^{\mathscr{C}} \in\left\{A_{n / c}, S_{n / c}\right\}$. Then by Lemma 7.2, one of the following holds: $G$ is quasiprimitive, $G$ is of Almost Simple Quotient type and $K$ is regular, or $c=3$ and $n=15$. In the third case, $m(G) \geq 2$ as $G$ acts faithfully on $\Omega$, and hence $m(G) \geq(\sqrt{15}-1) / 2$. If $G$ is quasiprimitive, then by [10, Theorem 7.2], either $m(G) \geq(\sqrt{n}-1) / 2$ or $G$ contains $A_{n}$. Now we turn to the case where $G$ is of Almost Simple Quotient type with a regular plinth. Note that $G$ is permutationally isomorphic to a subgroup of $\mathrm{Hol}\left(A_{n / c}\right)$ acting naturally on $A_{n / c}$, and so by Theorem 7.4, $m(G) \geq(n / c) ! / 2-(n / c-2) ! \geq(\sqrt{n}-1) / 2$.

\section{Quotient actions of innately transitive groups}

If $G$ acts quasiprimitively on $\Omega$, then the kernel $E$ of the action of $G$ on a $G$-invariant partition $\mathscr{B}$ of $\Omega$, is an intransitive normal subgroup of $G$. So we must have that $E=1$ and hence $G^{\mathscr{B}}$ is quasiprimitive and isomorphic to $G$. However, the quasiprimitive types for $G^{\Omega}$ and $G^{\mathscr{B}}$ may be different. The possible types for $G^{\Omega}$ and $G^{\mathscr{B}}$ were determined by Cheryl Praeger in [7, Theorem 1], which we recast below.

THEOREM 9.1 (Praeger). Let $G$ be an imprimitive quasiprimitive permutation group of type $X$ on a finite set $\Omega$, and let $\mathscr{B}$ be a non-trivial $G$-invariant partition of $\Omega$. Then $G^{\mathscr{B}}$ is quasiprimitive of type $X^{\mathscr{B}}$, where $X^{\mathscr{B}} \in\{A S, S D, C D, P A\}$, $X \in\{A S, S D, C D, T W, P A\}$, and the $\left(X, X^{\mathscr{B}}\right)$-entry in the Quotient Action Matrix in Table 2 is the symbol $\checkmark$. Conversely, if the symbol $\checkmark$ occurs in the $(X, Y)$-entry, then there exists a finite imprimitive quasiprimitive group of type $X$ with a non-trivial quotient action of type $Y$.

TABLE 2. Quotient Action Matrix

$\begin{array}{ccccc} & A S & S D & C D & P A \\ A S & \checkmark & \times & \times & \times \\ S D & \times & \times & \checkmark & \times \\ C D & \times & \times & \checkmark & \times \\ T W & \times & \checkmark & \checkmark & \checkmark \\ P A & \times & \checkmark & \checkmark & \checkmark\end{array}$


Now if instead $G$ is innately transitive on $\Omega$, it is not necessarily true this time that the kernel of the action of $G$ on $\mathscr{B}$ is trivial. We generalise Praeger's theorem below.

THEOREM 9.2. Let $G$ be an imprimitive innately transitive permutation group of type $X$ on a finite set $\Omega$, let $H$ be a finite permutation group on set $\Gamma$, and let $(\theta, \mu)$ be a faithful permutational transformation from $G^{\Omega}$ to $H^{\Gamma}$. Then $(G) \theta$ is innately transitive on $(\Omega) \mu$ of type $Y$, where $X, Y \in\{A S, A S Q, T Q, S D, C D, P A, D Q, P Q\}$, and the $(X, Y)$-entry in the Quotient Action Matrix in Table 3 is the symbol $\checkmark$. Conversely, if the symbol $\checkmark$ occurs in the $(X, Y)$-entry, then there exists an innately transitive group of type $X$ with a quotient action of type $Y$.

TABLE 3. Quotient Action Matrix

$\begin{array}{ccccccccc} & A S & A S Q & T W & S D & C D & P A & D Q & P Q \\ A S & \checkmark & \times & \times & \times & \times & \times & \times & \times \\ A S Q & \checkmark & \checkmark & \times & \times & \times & \times & \times & \times \\ T W & \times & \times & \checkmark & \checkmark & \checkmark & \checkmark & \times & \times \\ S D & \times & \times & \times & \checkmark & \checkmark & \times & \times & \times \\ C D & \times & \times & \times & \times & \checkmark & \times & \times & \times \\ P A & \times & \times & \times & \checkmark & \checkmark & \checkmark & \times & \times \\ D Q & \times & \times & \times & \checkmark & \checkmark & \checkmark & \checkmark & \times \\ P Q & \times & \times & \times & \checkmark & \checkmark & \checkmark & \times & \checkmark\end{array}$

ProOF. First recall that $(G) \theta$ is innately transitive with plinth $(K) \theta$ and $(K) \theta$ is isomorphic to $K$. So $(K) \theta$ is simple if and only if $K$ is simple, and hence $X \in\{A S, A S Q\}$ if and only if $Y \in\{A S, A S Q\}$. By Lemma 5.2, if $(K) \theta$ is regular then $K$ is regular. Hence if $Y \in\{T W, D Q, P Q\}$ then $X \in\{T W, D Q, P Q\}$. We also know that if $G^{\Omega}$ is primitive (respectively quasiprimitive) then $(G) \theta^{(\Omega) \mu}$ is primitive (respectively quasiprimitive). Since $G^{\Omega}$ is imprimitive, $G^{\Omega}$ and $(G) \theta^{(\Omega) \mu}$ are not of Abelian Plinth, Holomorph of a Simple Group, or Holomorph of a Compound Group type. So far we have ruled out values of $(X, Y)$ indicated in Table 4.

So suppose now that $K$ is non-simple, and let $\alpha \in \Omega$ and $\Delta=\alpha^{\mathrm{ker} \theta}$. Note for all $x \in \operatorname{ker} \theta$, that $\left(\alpha^{x}\right) \mu=(\alpha) \mu^{(x) \theta}=(\alpha) \mu$ and hence $(\Delta) \mu=\{(\alpha) \mu\}$. So for all $y \in K_{\Delta}$, we have $(\alpha) \mu^{(y) \theta}=\left(\alpha^{y}\right) \mu \in(\Delta) \mu=\{(\alpha) \mu\}$ and hence $(K) \theta_{(\alpha) \mu}$ contains $\left(K_{\Delta}\right) \theta$. Now by comparing orders (and using Lemma 7.1) we see that $(K) \theta_{(\alpha) \mu}$ is equal to $\left(K_{\Delta}\right) \theta$ :

$$
\begin{aligned}
\left|(K) \theta_{(\alpha) \mu}\right| & =|(K) \theta| /|(\Omega) \mu|=|(K) \theta||\operatorname{ker} \theta| /|\Omega| \\
& =|K||| K_{\Delta}: K_{\alpha}|/| K: K_{\alpha}|=| K_{\Delta} \mid .
\end{aligned}
$$

In the case that $X \in\{D Q, P Q\}$, we have that $G$ is not quasiprimitive and hence $\mathrm{C}_{G}(K)$ is nontrivial. Thus $K_{\triangle}$ is nontrivial and $(K) \theta$ is not regular. So if $X \in\{D Q, P Q\}$, then 
TABLE 4. Impossibilities thus far.

$\begin{array}{ccccccccc} & A S & A S Q & T W & S D & C D & P A & D Q & P Q \\ A S & \checkmark & \times & \times & \times & \times & \times & \times & \times \\ A S Q & & \checkmark & \times & \times & \times & \times & \times & \times \\ T W & \times & \times & \checkmark & & & & \times & \times \\ S D & \times & \times & \times & \checkmark & & & \times & \times \\ C D & \times & \times & \times & & \checkmark & & \times & \times \\ P A & \times & \times & \times & & & \checkmark & \times & \times \\ D Q & \times & \times & & & & & \checkmark & \\ P Q & \times & \times & & & & & & \checkmark\end{array}$

$Y \notin\{T W, D Q, P Q\}$. The only cases we must rule out now are $(X, Y)=(S D, P A)$, $(X, Y)=(C D, S D)$, and $(X, Y)=(C D, P A)$.

Suppose $K_{\alpha}$ is a subdirect subgroup of $K$. By the proof of [1, Proposition 5.5], $K_{\alpha}$ is self-normalising and hence $K_{\alpha}=K_{\Delta}$ (by Lemma 7.1). Thus it follows that (K) $\theta_{(\alpha) \mu}$ is a subdirect subgroup of $(K) \theta$. So if $X \in\{S D, C D\}$ then $Y \in\{S D, C D\}$, and $(X, Y) \notin\{(S D, P A),(C D, P A)\}$. Finally, suppose $(X, Y)=(C D, S D)$. Then $\left(K_{\Delta}\right) \theta$ is a full diagonal subgroup of $(K) \theta$. So in particular, $\left(K_{\Delta}\right) \theta$ is isomorphic to each simple direct factor of $(K) \theta$. This implies that $K_{\Delta}$ is isomorphic to a simple direct factor of $K$, and hence $K_{\Delta}$ is simple. However, $K_{\alpha}$ is a proper nontrivial normal subgroup of $K_{\Delta}$-a contradiction. Therefore $(X, Y) \neq(C D, S D)$ in this case.

This analysis verifies that the crossed entries in Table 3 are correct. Next we prove that examples exist for each checked entry in Table 3. By Praeger's Theorem, we have examples for most of our table except the cases $X=A S Q, P Q, D Q$. We complete the table with the following examples.

$X=A S Q$. Let $G$ be an innately transitive group of Almost Simple Quotient type (for an example, see Example 3) and let $\mathscr{C}$ be the set of orbits of the centraliser of the plinth of $G$. Then $G^{\mathscr{C}}$ is quasiprimitive of Almost Simple type as the plinth of $G^{\mathscr{C}}$ is isomorphic to the plinth of $G$. So we have examples where $(X, Y)=(A S Q, A S)$.

$X=P Q$. Let $G$ be an innately transitive group on a set $\Omega$ with plinth $K$, and suppose $G$ is of Product Quotient type. Let $\mathscr{C}$ be the orbits of $\mathrm{C}_{G}(K)$. Then the stabiliser $K_{\Delta}$ of an element $\Delta \in \mathscr{C}$ is not a subdirect subgroup of $K$. Therefore $G^{\mathscr{C}}$ is quasiprimitive of Product Action type. Now consider the example given in Example 4 for $X=P A$. If one chooses $S=1$, then $G=\operatorname{Diag}\left(R^{k}\right) \times(T \mathrm{wr} A)$ is innately transitive on $\Omega$ of Product Quotient type. The centraliser of $K$ in $G$ is $C$ and $G^{\mathscr{C}}$ is precisely the quasiprimitive group of Product Action type given in [7, Example 5]. In this example, Praeger shows that $G^{\mathscr{C}}$ has quasiprimitive quotient actions of Simple Diagonal and Compound Diagonal type. Hence our group $G$ also has quotient actions of Simple Diagonal and Compound Diagonal type. 
$X=D Q$. Let $G$ be an innately transitive group on a set $\Omega$ with plinth $K$, and suppose $G$ is of Diagonal Quotient type. Let $\mathscr{C}$ be the orbits of $\mathrm{C}_{G}(K)$. Then by Lemma 1.1 (2) (or Lemma 7.1) the stabiliser $K_{\Delta}$ of an element $\Delta \in \mathscr{C}$ is a subdirect subgroup of $K$. Therefore $G^{\mathscr{C}}$ is quasiprimitive of Diagonal type. The innately transitive group $G$ in Example 2 is an example where $G^{\mathscr{C}}$ is of Diagonal type. In this example, if $m=1$ then $G^{\mathscr{C}}$ is of Simple Diagonal type, otherwise $G^{\mathscr{C}}$ is of Compound Diagonal type. We will show now that there is an example where $\left(X, X^{\mathscr{B}}\right)=(D Q, P A)$. Take Example 2 where $G=K \rtimes\left[\left(A \times S_{k / m}\right)\right.$ wr $\left.S_{m}\right], C=\operatorname{Diag}\left(T^{k / m}\right)$, and $\mathrm{C}_{G}(K)=C^{m}$ (in the left regular representation of $K$ ). Let $M$ be a proper nontrivial subgroup of $C^{m}$ in the right regular representation of $K$. So $M$ is a diagonal subgroup of $K$, but is not subdirect. Now $G$ acts on $\mathscr{B}=\left[G: G_{1} M\right]$, where $G_{1}$ is the stabiliser in $G$ of the identity element, and induces an innately transitive group on $\mathscr{B}$ with plinth $K$. Since $M$ is a point stabiliser for the plinth in this action, and $M$ is nontrivial and not subdirect, it follows that $G^{\mathscr{B}}$ is of Product Action type.

Therefore, examples exist for each checked entry in Table 3.

\section{References}

[1] J. Bamberg and C. E. Praeger, 'Finite permutation groups with a transitive minimal normal subgroup', Proc. London Math. Soc. (3) 89 (2004), 71-103.

[2] A. Bochert, 'Ueber die Zahl der verschiedenen Werthe, die eine Function gegebener Buchstaben durch Vertauschung derselben erlangen kann', Math. Ann. 65 (1889), 584-590.

[3] J. D. Dixon and B. Mortimer, Permutation groups (Springer, New York, 1996).

[4] D. R. Heath-Brown, C. E. Praeger and A. Shalev, 'Permutation groups, simple groups, and sieve methods', Israel J. Math., to appear.

[5] M. W. Liebeck, C. E. Praeger and J. Saxl, 'On the O'Nan-Scott theorem for finite primitive permutation groups', J. Austral. Math. Soc. 44 (1988), 389-396.

[6] C. E. Praeger, 'An O'Nan-Scott theorem for finite quasiprimitive permutation groups and an application to 2-arc transitive graphs', J. London Math. Soc. (2) 47 (1993), 227-239.

[7] - 'Quotients and inclusions of finite quasiprimitive permutation groups', J. Algebra 269 (2003), 329-346.

[8] C. E. Praeger, C. H. Li and A. C. Niemeyer, 'Finite transitive permutation groups and finite vertextransitive graphs', in: Graph symmetry (Montreal, PQ, 1996) (Kluwer Acad. Publ., Dordrecht, 1997) pp. 277-318.

[9] C. E. Praeger and J. Saxl, 'On the orders of primitive permutation groups', Bull. London Math. Soc. 12 (1980), 303-307.

[10] C. E. Praeger and A. Shalev, 'Bounds on finite quasiprimitive permutation groups', J. Austral. Math. Soc. 71 (2001), 243-258.

School of Mathematics and Statistics

University of Western Australia

35 Stirling Highway

Crawley WA 6009

Australia

e-mail: john.bam@maths.uwa.edu.au 